rewards, both personal and professional, of scientific research. He treats the reader to a behind-the-scenes look at the jealousy, backstabbing, ambition and embellishment that comes with the territory of the modern scientific quest to halt or turn back the hands of time. In the end, Hall makes it clear that although today's immortalists may be whispering optimistically about their own immortality as they take their own last breath, there is a glimmer of hope that the true fountain of youth could be just over the horizon.

Merchants of Immortality is a remarkable book that is a must-read in the world of science and medicine. And lay readers will be interested in this revealing story about the odd mixture of science, politics and ethics, and how they all come together in the modern quest for immortality.

S. Jay Olshansky is at the School of Public Health,

University of Illinois at Chicago, Chicago,

Illinois, USA.

\section{Ecology you can count on}

\section{Population Ecology: First \\ Principles}

by John H. Vandermeer \& Deborah E.

Goldberg

Princeton University Press: 2003. 296 pp.

$\$ 75, \mathfrak{E} 52$ ( $h b k$ ); $\$ 35, \mathfrak{£} 24.95$ (pbk)

\section{Alan Hastings}

A mathematical and conceptual approach to population ecology - the study of the dynamics of animals and plants in nature - can be traced back to work early in the twentieth century. The fundamentals were developed by a number of scientists: Alfred J. Lotka, Vito Volterra and G. F. Gause worked on the dynamics of single and interacting populations; A. J. Nicholson and V. A. Bailey studied host-parasitoid dynamics; Lotka and P. H. Leslie did the early work on age-structured populations; J. G. Skellam did the same for spatial dynamics; and W. O. Kermack and A. G. McKendrick, and then Norman T. J. Bailey and others, worked on the dynamics of diseases and epidemics.

These innovators set the stage for recent advances in the understanding of the dynamics of ecological populations, and in applied areas such as the management of renewable resources, conservation biology and understanding the population dynamics of diseases. Progress in all these areas has come from a tight coupling of mathematical theory with biology, and students and ecologists need a good grounding in these areas. How well does the book Population Ecology succeed in developing the field from a set of first principles, and who is most likely to profit from reading it?

Research based on first principles in population ecology depends, at the very least, on a thorough understanding of basic calculus and linear algebra, and arguably needs more advanced techniques as well, such as stochastic processes and partial differential equations. So it is important how the book treats these areas, and what knowledge it assumes on the part of the reader. The stated assumption is that readers are familiar with calculus but may not have seen the required results in linear algebra - there is a brief appendix summarizing the major results for linear algebra, but not for calculus. I think that to follow the mathematical development, readers will need a good to outstanding working knowledge of calculus and be comfortable with mathematical manipulations.

In some respects, the chapter on dynamics emerges as one of the strongest parts of the book; in others, it is the weakest. It is a truly beautiful exposition of nonlinear dynamics at a relatively elementary level, but unfortunately it is almost devoid of biology. And in population ecology, where mathematics and biology are intimately connected, it is important that these disciplines are well integrated. The rest of the book does this much better, helping readers to understand how both need to be used to get at the heart of population ecology. The chapter on space and metapopulations goes further, by explaining the importance of a statistical approach to understanding spatial pattern, and in so doing it emphasizes the role of model formulation.

As is typical in ecology, many mathematical arguments are presented graphically. Some of these are well done, for example in the chapter on competition models, but some are confusing, such as those illustrating the components of predator-prey interactions. In a few places, such as the chapter on life histories, a more graphical development would have been welcomed.

Overall, the book provides a solid foundation for further study in population ecology, and provides good coverage of all the fundamental areas of population ecology outlined above. But as a textbook, or for individual study, there is room for improvement. The lack of problems to solve is a big shortcoming, as mathematical developments are best understood by doing, rather than by merely reading. And readers who are not advanced mathematically might find several places where the mathematical development is too terse, for example in the chapter on projection matrices. Also, the book doesn't use many different biological examples to bolster an argument, just a few choice studies (very few in the chapter on competition). Yet the overall concept of emphasizing first principles in population ecology is very appealing, and readers who understand the material presented will be able to cope with most current research in population ecology.

Alan Hastings is in the Department of

Environmental Science and Policy, University of California, Davis, California 95616, USA. He is the author of Population Biology: Concepts and Models.

\section{Petal power}

Illustrations of orchids, such as those of Eriochilus cucullatus shown here, are normally found in historic texts predating modern photography. But Princeton University Press has just published the first in a series of books on the Orchids of Australia, each containing new illustrations of some 150 species by artist John J. Riley. Accompanied by descriptive text by Riley and David P. Banks, these books are intended to provide a visual diagnostic reference for orchid enthusiasts, as well as a feast for admirers of botanical illustration as art.

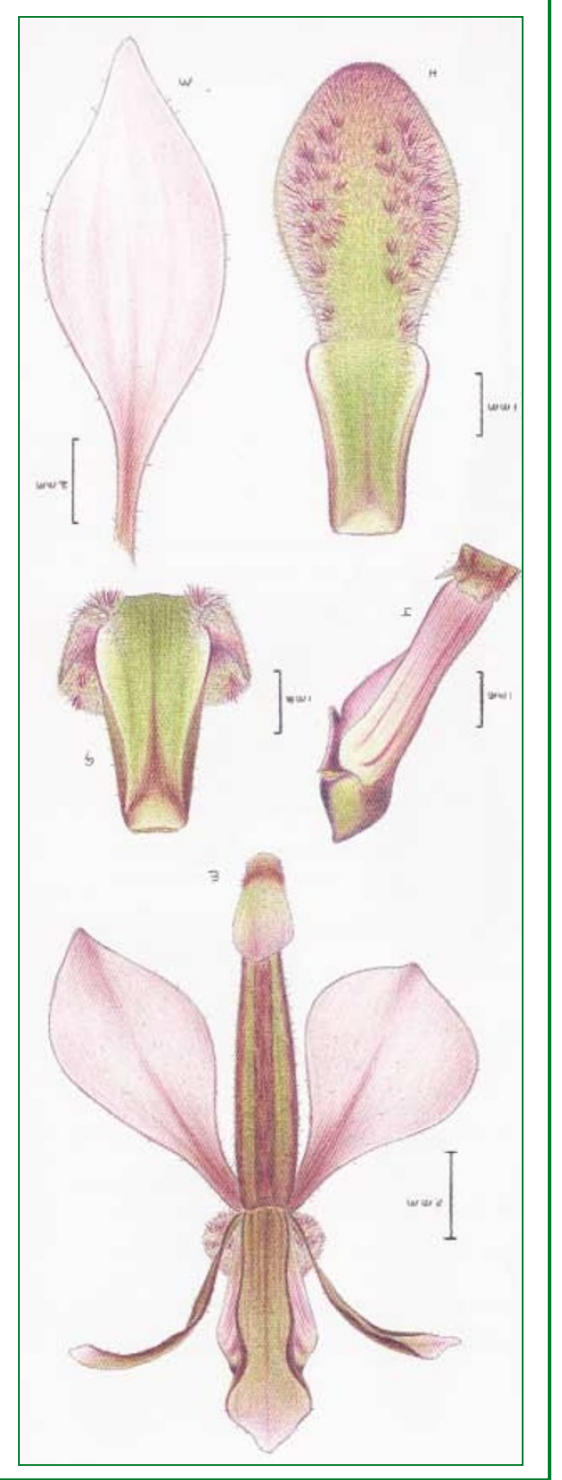

